\title{
STUDY ON TACTILE PERCEPTION OF SHAPE FOR EFFECTIVE TACTILE DISPLAY
}

\author{
Myoung-Jong Yoon, Kee-Ho Yu, Tae-Kyu Kwon and Nam-Gyun Kim
}

\author{
School of Mechanical \& Aerospace Systems Engineering \\ Chonbuk National University, Chonju, 561-756, Korea
}

\begin{abstract}
The experiment of the shape recognition for a tactile display is carried out and analysed in this paper. The purpose of this research is the development of a tactile stimulator array to represent the obstacle information for the visually impaired to walk safely. As a first step of this research, we investigate the characteristics of tactile stimuli and design the experimental system for the physiological characteristic test based on the investigated results. In order to determine the appropriate and optimal range of the actuating frequency and displacement for the tactile stimulation array, the psychophysical experiment for the threshold of perception and recognition ability of multi-stimulation with some shapes are carried out by the estimation of the subject group. As a result the appropriate tactile stimulus intensity and frequency of the tactile stimulation array to recognize arbitrary line shapes effectively are discussed and derived. Copyright () 2005 IFAC
\end{abstract}

Keywords: Shape Recognition, Tactile Display, Tactile Stimulator, Obstacle, Array, Vibration, Threshold

\section{INTRODUCTION}

Many of blind persons use the white cane or guide dog. The white cane is a successful and widely used travel aid for the blind. However, users must be trained in the use of the white cane for a period of over 100 hours. The guide dog is expensive, difficult to train, and sometimes unfriendly to other pedestrians (Shoval, et al., 2003). Therefore, the development of an alternative engineering system is strongly needed. For the visually impaired some engineering system such as artificial retina, guide robot, ETA(Electronic Travel Aid) have been developed, but in the sense of practical use, those research and development are not fully satisfied.

In the case of ETAs, a fundamental shortcoming with all ETAs based on acoustic feedback is their interference (called "masking") with sound cues from the environment and reducing the blind person's ability to hear these essential cues (Shoval, et al., 2003). So, this research focuses on the tactile feedback for the transfer of obstacle information in ETA.

The existent tactile stimulators are divided by pneumatic, vibrotactile, electrotactile stimulation etc
(Sato, et al., 1991; Watanabe, et al., 1995; Kontarinis, et al., 1995; Konyo, et al., 2000). The touch senses of the human are poor at determining absolute quantities but very sensitive to changes (Russell, 1990). So. We are interested in the vibrotactile stimulation. This kind of device is compact and light, and this feature would be helpful for the portability needed in the guide system for the blind.

To display the obstacle information such as position, size, shape, and movement of the obstacle, an arraytype tactile stimulator is needed. To make an effective array type tactile stimulator some main parameters, like as, the displacement and frequency of the vibration, and the density and numbers of tactels should be considered.

The objective of this research is the development of a portable tactile stimulator to display some obstacle information for the visually impaired pedestrian. As a first step of this research, in this paper, we investigate the characteristics of the tactile stimuli and experiment the perception characteristics by the subject group based on the investigated results. In order to determine the optimal parameters for an array type of tactile stimulator, the experiment for the 
threshold and perception ability of multi-stimulation with some shape were carried out. It is important first of all to decide the optimal parameters before fabrication of a tactile stimulator. In the experiment, the perception threshold to the tactile stimulus intensity is obtained using the experimental set-up prepared for the physiological perception test. According to the obtained experimental result, the appropriate tactile stimulus intensity for the recognition of multi-stimuli with some shape is determined. Also the psychophysical experiments of multi-stimuli recognition with arbitrary shape are carried out.

In Section 2, we investigate the characteristics of the tactile stimuli. In Section 3, we illustrate an outline of the experimental set-up. In section 4 and 5, we describe the psychophysical experiment for threshold and recognition of multi-stimulation with some shape respectively.

\section{CHARACTERISTICS OF TACTILE STIMULI}

To be able to design a tactile stimulator understanding of the nerve activities is essential - in particular the present physiological knowledge of the nerves contributing to the sensations must be understood.

Human skin has a number of sense receptors of elementary sensations such as touch, pressure, vibration, pain, temperature, etc. The main elementary sensations given by mechanical stimuli are pressure sense and vibration sense. Mechanoreception is concerned with the detection of mechanical stimuli at the skin and comprises four qualities; the sensations of pressure, touch, vibration, and tickle, detected by a series of nerve endings including Merkel disks, Ruffini endings, Meissner corpuscles, and Pacini corpuscles, each of which have different perception characteristics. Four different kinds of sensors register deformation of the skin caused by contact with external objects. Meissner corpuscles and Merkel disks lie close to the skin surface and have high spatial resolution. In the fingerprint skin of the hand Meissner corpuscles are located between the papillary ridges of the dermis, while the Merkel disks are located at the ends of these ridges. Pacini corpuscles and Ruffini endings are embedded deep in the skin and hence there receptive fields are much broader. In addition, there are free nerve endings with a high threshold which respond to painful and potentially harmful stimuli, such as pricking with a sharp needle. The concerning sensory receptors are shown in Fig. 1 (Konyo, et al., 2000; Russell, 1990; Galdwell, et al., 2000; Bolanowski, et al., 1998; Guyton, et al., 2002).

Mechanoreceptive nerve fibers can be categorized by two criteria: the size of their active areas (receptive field), and the speed of their adaptation to static stimuli. Mechanoreceptive nerve fibers with small receptive fields are called type I units, while those with large fields are called type $\Pi$. Nerve fibers that respond to static stimuli are called SA (slowly adapting), while those with no static response are called FA or RA (fast or rapidly adapting). Based on morphological observation (receptive field characteristics, adaptive properties of the fibers to stepwise indentation and frequency response to sinusoidal vibration), attempts have been made to associate SA I with Merkel disks, SA II with Ruffini endings, FA I with Meissner corpuscles, and FA II with Pacini corpuscles. Merkel disks form 25 percent of the receptors in the hand and have a disk-like nerve ending. These receptors respond best to pressure, but can also provide vibration information. These receptors have respond bandwidth in 2-32Hz. Ruffini endings have a fusiform structure and make up approximately 19 percent of the hand receptors. They detect pressure and skin shear as well as thermal changes. These receptors have respond bandwidth in $1-16 \mathrm{~Hz}$. Meissner corpuscles represent over 40 percent of the hand tactile receptors. Since they move with the ridges of the skin, these receptors can best detect the movement across the skin and function as velocity detectors. These receptors have respond bandwidth in $8-64 \mathrm{~Hz}$. Pacini corpuscles are the largest of the skin corpuscle receptors and represent 13 percent of the hand receptors. They detect light touch as well as vibrations and function as acceleration detectors. These receptors have respond bandwidth in 64$400 \mathrm{~Hz}$ widely. Also, those respond to very small stimulus displacement $(\mu \mathrm{m})$ (Russell, 1990; Galdwell, et al., 2000; Bolanowski, et al., 1998).

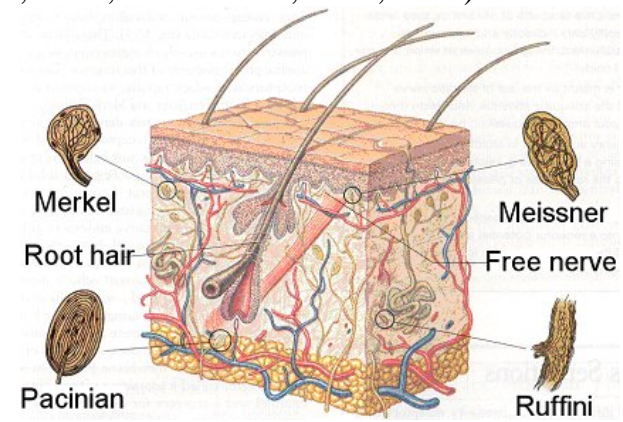

Fig. 1. The cutaneous sensory receptors (Guyton, et al., 1998).

\section{DESCRIPTION OF EXPERIMENTAL APPARATUS}

As shown in Fig. 2, sinusoidal, vibratory displacements of the stimulation array are produced with a TIRAvib S511 vibrator under computer control (dSPACE1103 DSP system) and applied to the fingertip. The displacements are produced around a static indentation of $1 \mathrm{~mm}$ and monitored with a CCD laser displacement sensor (LK010, KEYENCE) that senses the displacement of the moving element of the vibrator. The resolution of the displacement sensor is $0.1 \mu \mathrm{m}$, measuring range is $2 \mathrm{~mm}$, and linearity is $\pm 0.25 \%$. Fig. 3 shows the device for stimulation array that consists of pin array, base for the pin array, and contact surface. 


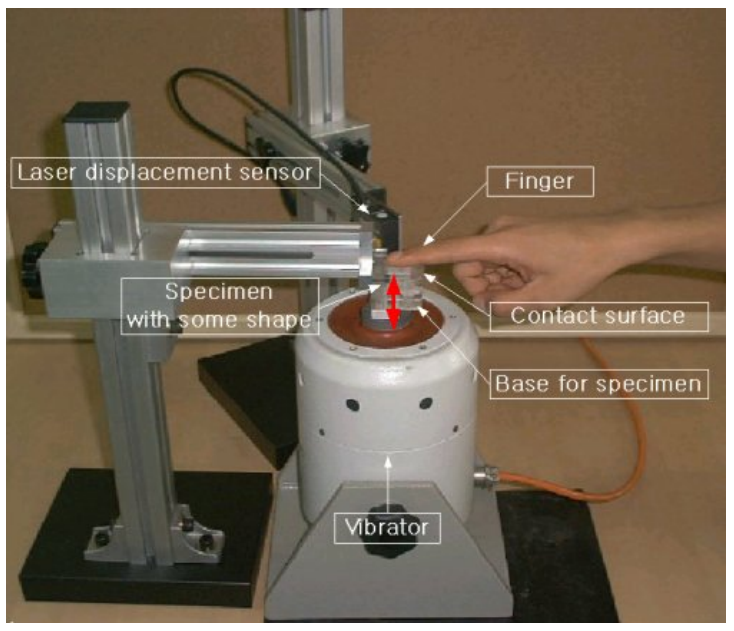

Fig. 2. Experimental apparatus for physiological perception test.

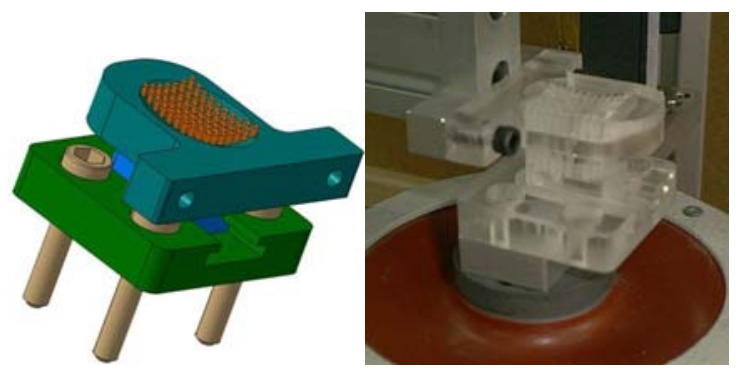

Fig. 3. Integrated device for stimulation array.

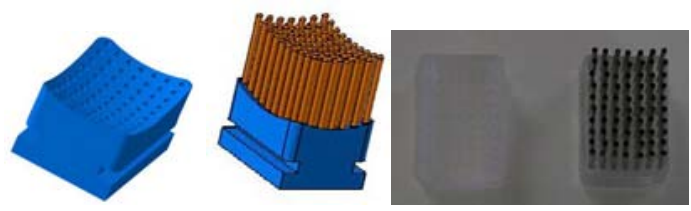

Fig. 4. Sample pin array for stimulation.

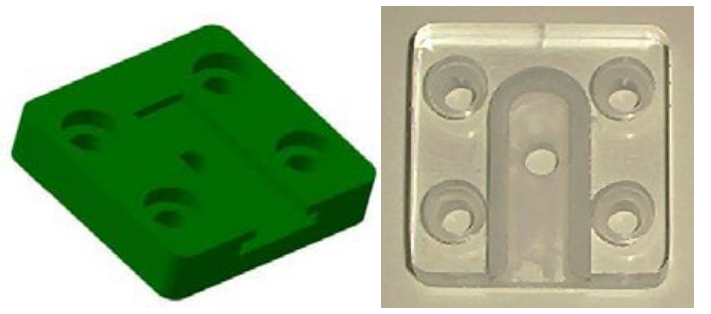

(a)

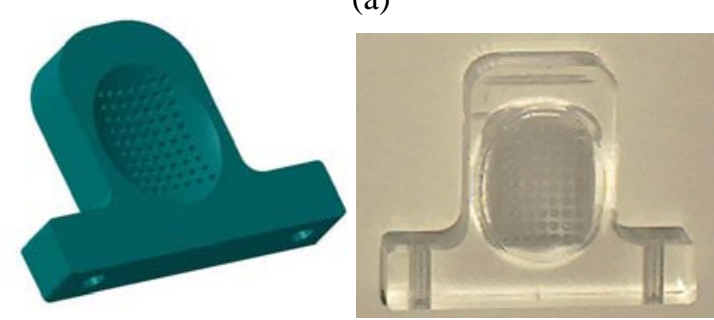

(b)

Fig. 5. (a) Base frame (b) Contact surface.

Fig. 4 shows a sample of pin array. As steel pins are fixed in holes with pin array, we can make stimulation arrays with variable shapes such as triangle, square, circle, etc. To enhance the contact state closely, the upper surface of pin array is designed to have the bowl shape like as the surface of a fingertip.

The number of available tactels(steel pins) is $7 \times 10$. The tactel spacing is $2 \mathrm{~mm}$, the diameter of each tactel is $1 \mathrm{~mm}$, and length of tactel is $15 \mathrm{~mm}$. The effective stimulation area properly matched for the index finger is $15 \mathrm{~mm} \times 20 \mathrm{~mm}$.

To easily change the stimulation array and fix it to vibrator, the base frame is designed as shown in Fig. 5 (a). Fig. 5 (b) shows the contact surface that supports the finger maintaining close contact. The diameter of the each hole in contact surface is 1.4 $\mathrm{mm}$, so that, the margin of $0.2 \mathrm{~mm}$ is exist between the edge of the hole and actuating tactel for the stimulation without interference.

\section{EXPERIMENT FOR THRESHOLD OF TACTILE PERCEPTION}

\subsection{The Objective of Experiment}

In order to investigate the threshold of multisimulation with some shape, this experiment was carried out. The threshold of tactile stimulus intensity within various frequency bandwidth was investigated in some papers (Bolanowski, et al., 1988; Johansson, et al., 1982; Yoon, et al., 2004). However, those papers showed the experiment results using only one tactel. So those experiment results are not useful for array-type tactile stimulator that will be developed for the purpose of displaying some shape. In this section the experiment for the threshold of multistimulation with some shape was carried out.

Through this experiment, we try to find the appropriate frequency bandwidth and intensity (displacement) of vibrating stimuli for recognition of some shape in human finger.

\subsection{The Method of Experiment}

Fig. 6 shows the configuration of the experimental set-up for threshold of multi-stimulation with some shape. The vibration of sine wave generated by the vibrator (TIRAvib S511) is applied to a fingertip, and the vibration is measured dynamically by the CCD laser displacement sensor (LK010, KEYENCE), and then the output signal of the laser sensor is processed in the DSP system (dSPACE 1103) of personal computer.

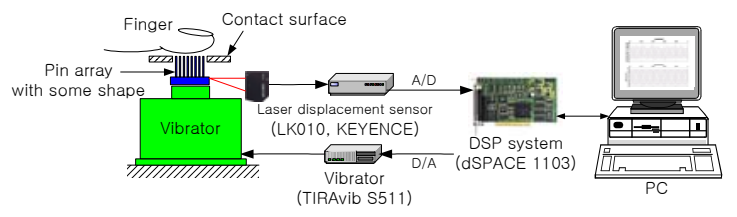

Fig. 6. Configuration of experimental set-up. 
This experiment was performed on healthy adults without a previous notice about the objective of the experiment, so that, it is possible to prevent the effect of learning. A total of ten subjects, two female (age 23,25 ) and eight males (age 25-32) were used in the experiment. The sinusoidal vibratory stimuli with some shape were delivered to the subject's fingertip using the vibrator. The subjects are requested to judge whether they recognized the stimulated shape or not and answered the recognized shape. The minimum stimulus intensity (displacement) in which the stimulated shape and subject's answer are the same was recorded. The minimum stimulus intensity is defined as the thresholds of perception. The thresholds were measured for each of the frequencies of the test stimulus.

In this experiment, we used pin array with some shape such as triangle(Fig. 7 (a)), square(Fig. 7 (b)), and circle(Fig. 7 (c)) which are the basic shapes among arbitrary shapes. The pin arrays used in the experiment are shown in Fig. 7.

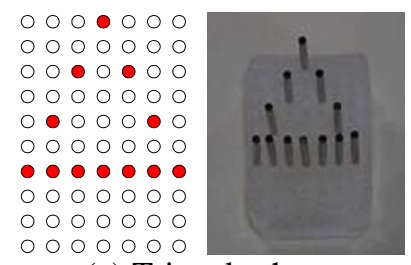

(a) Triangle shape

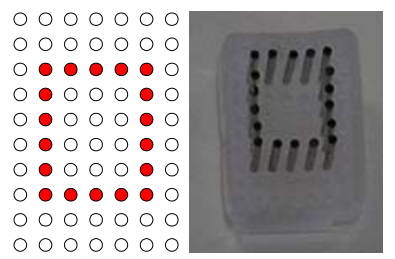

(b) Square shape

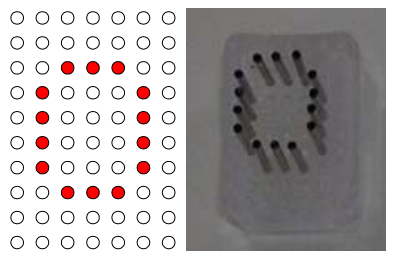

(c) Circle shape

Fig. 7. Pin array for test.

\subsection{Perception Threshold to One Tactel Stimulation}

Fig. 8 shows the threshold of tactile stimulus intensity within a frequency $5-500 \mathrm{~Hz}$ and at various stimulus intensity using only one tactel. In this result, the threshold means the minimum stimulus intensity that the subject recognizes stimulation. The results are the averages of ten subjects. The error bars signify the standard error of the means. The presented results indicate that vibratory stimuli can be recognized at frequencies between $5 \mathrm{~Hz}$ and $500 \mathrm{~Hz}$, the threshold-frequency characteristic shows decreased slope for the low range. The general shape of the characteristic is similar to Bolanowski's result
(Bolanowski, et al., 1988). In generally, human skin is more sensitive to high frequency stimulus than low frequency one in same stimulus intensity (displacement). However, the sensitivity of the perception is not improved any more in the high frequency range of over $400 \mathrm{~Hz}$. From the experiment the minimum threshold is about $1 \mu \mathrm{m}$ at $250 \mathrm{~Hz}$.

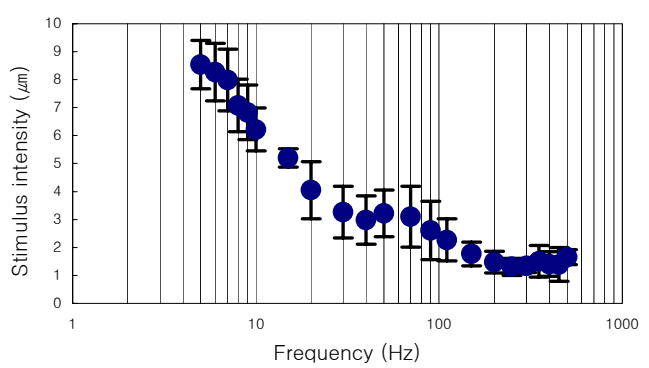

Fig. 8. Threshold of perception to stimulation by one tactel.

\subsection{Perception Threshold to Line Shape Tactile Stimulation}

The experiment result using only one tactel is not useful for array-type tactile stimulator that will be developed for the purpose of displaying some shape. So, the experiment for threshold of multi-stimulation with some shape should be carried out. The perception threshold of tactile stimulus intensity was obtained in the frequency range of 1 to $50 \mathrm{~Hz}$ using experimental pin array and the result is shown in Fig. 9. The results are the averages of ten subjects. The error bars signify the standard error of the means.

The results mean the minimum displacement (intensity) to transfer the tactile stimulation of some line shape to a human fingertip in the frequency range of 1 to $50 \mathrm{~Hz}$. The mechanoreceptor that has function of shape detection is Merkel disks, and its respond bandwidth is 2-32Hz (Russell, 1990). Therefore, the stimulus frequency bandwidth of 1 to $50 \mathrm{~Hz}$ used in the experiment is reasonable. In the results, the similar aspect of the measured threshold is appeared although a little difference exists in each shape. The threshold-frequency characteristic is partially sloping for the stimulus frequency bandwidth in each result.

Comparing this result with the case of one tactel, the threshold of stimulus intensity has larger value than the case of only one tactel (Bolanowski, et al., 1988; Johansson, et al., 1982; Yoon, et al., 2004). It is clear that, since this experiment is carried out for recognition of multi-stimuli with some shape, the threshold of stimulus intensity became larger comparatively. However, for the exact relation between the number of tactels and the threshold of stimulus intensity, it is needed to study further.

According to the obtained experimental results as shown in Fig. 9, the appropriate tactile stimulus intensity for the recognition of multi-stimuli with some shape was determined in the frequency range of 
1 to $50 \mathrm{~Hz}$. In the obtained experimental results, the threshold of stimulus intensity is about $120 \mu \mathrm{m}$ at $1 \mathrm{~Hz}$. And it is almost maximum threshold in the stimulus frequency bandwidth of 1 to $50 \mathrm{~Hz}$. Therefore, in the above frequency range, the displacement of $120 \mu \mathrm{m}$ or more is recommended as a tactile stimulus intensity.

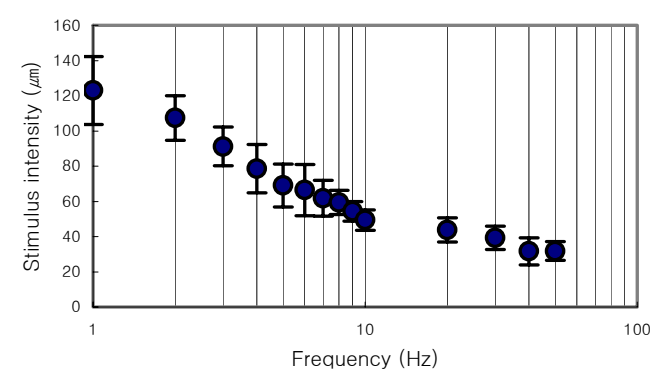

(a) Triangle shape

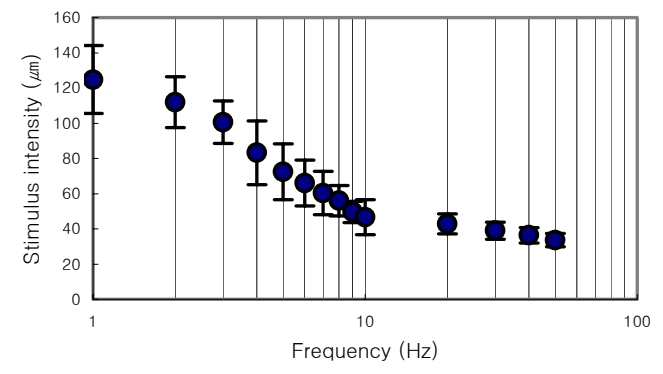

(b) Square shape

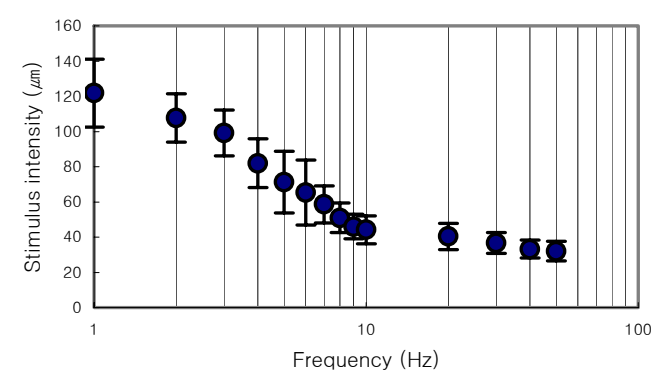

(c) Circle shape

Fig. 9. Perception threshold to stimulation by multitactels with some shape.

\section{EXPERIMENT FOR TACTILE PERCEPTION OF LINE SHAPES}

\subsection{The Objective and Method of Experiment}

The objective of this experiment is the investigation of the perception ability of multi-stimulation with some shape to the variable stimulus intensity and frequency. In this experiment, shapes of the used pin array for multi-stimulation are the same as the previous experiment.

This experiment was performed on 10 healthy adults without a previous notice about the objective of the experiment also, so that, the condition of this experiment is the same as the previous experiment. For the shape recognition, this experiment was carried out at the determined tactile stimulus intensity. In the experiment, the subjects are requested to answer the shape what he recognized among the shape of triangle, square, and circle. In the first experiment, the tactile stimulus frequency is fixed at $20 \mathrm{~Hz}$ and the stimulus intensity is changed within 20-200 $\mu \mathrm{m}$. And as the second experiment the tactile stimulus intensity is fixed at $100 \mu \mathrm{m}$ and the stimulus frequency is changed within $1-50 \mathrm{~Hz}$.

\subsection{The Experimental Results and Analyses}

Fig. 10 shows the experimental results of the correct answer rate by variable stimulus intensity at $20 \mathrm{~Hz}$. In the result, about $60 \%$, the correct answer rate is obtained at $20 \mu \mathrm{m}$ and it is increased about $100 \%$ according to intensity increase. At 20-80 $\mu \mathrm{m}$, the correct answer rate is increased rapidly. However, at $80-200 \mu \mathrm{m}$, it is increased gently. This means that the correct answer rate is not linear as increasing of the tactile stimulus intensity. At $80 \mu \mathrm{m}$, the correct answer rate is about $90 \%$, and this intensity is almost twice of the threshold. According to the result of Fig. 9 , the threshold of stimulus intensity is about $50 \mu \mathrm{m}$ at $20 \mathrm{~Hz}$. Therefore, to obtain the recognition rate more than $90 \%$, a double stimulus intensity of the threshold is recommended.

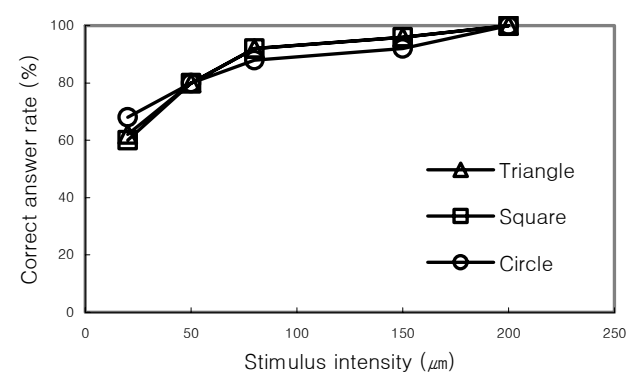

Fig. 10. Correct answer rate by variable stimulus intensity at the frequency of $20 \mathrm{~Hz}$.

Table 1 shows the experimental results of the correct answer rate by variable stimulus frequency at $100 \mu \mathrm{m}$. In the result, the correct answer rate is about $60 \%$ at $1 \mathrm{~Hz}$ and it is increased about 90\% according to frequency increase. This tendency could be explained by the previous experiment for the threshold of multi-stimulation in which the perception sensitivity is improved according to the frequency increase.

Comparing correct answer rates by shape, any particular differences are not appeared. But in the discrimination of square and circle shape, a little bias is shown in the correct answer rate. It seems that this bias is due to their similarity in shape. In the experiment result, at the stimulation of $50 \mathrm{~Hz}$ and $100 \mu \mathrm{m}$, the correct answer rate is about $95 \%$. This result is acceptable to recognize the stimulation with line shape. As a result we can see that the 
specification satisfying the above stimulation condition is required to make the effective tactile stimulation array.

Table 1 Correct answer rate by variable stimulus frequency at the intensity of $100 \mu \mathrm{m}$

\begin{tabular}{cccc} 
Answer & Triangle & Square & Circle \\
\hline Shape & 60 & 24 & 16 \\
Sriangle & 12 & 60 & 28 \\
Circle & 16 & 24 & 60 \\
\hline
\end{tabular}

(a) Frequency : $1 \mathrm{~Hz}$

\begin{tabular}{cccc} 
Answer & Triangle & Square & Circle \\
Shape & 68 & 16 & 16 \\
\hline Triangle & 8 & 72 & 28 \\
Square & 0 & 28 & 72 \\
Circle & 0 & \\
\hline
\end{tabular}

(b) Frequency : $5 \mathrm{~Hz}$

\begin{tabular}{cccc} 
Answer & Triangle & Square & Circle \\
Shape & 88 & 8 & 4 \\
Triangle & 0 & 88 & 12 \\
Square & 0 & 12 & 88 \\
Circle & 0 & \\
\hline
\end{tabular}

(c) Frequency : $10 \mathrm{~Hz}$

\begin{tabular}{cccc} 
Answer & Triangle & Square & Circle \\
Shape & 100 & 0 & 0 \\
Triangle & 0 & 96 & 4 \\
Square & 0 & 4 & 96 \\
Circle & 0 & \\
\hline
\end{tabular}

(d) Frequency: $50 \mathrm{~Hz}$

\section{CONCLUSION AND FUTURE WORK}

The purpose of this research is the development of a portable tactile stimulator to represent the obstacle information for the visually impaired pedestrian. As a first step of this research, in this paper, we investigated the perception characteristics of tactile stimuli in human skin from the previous reports and design the experimental system to find the optimal condition of the tactile display array based on the investigated results. In order to determine the optimal and appropriate parameters for an array type tactile stimulator, the experiment for the perception threshold and the recognition ability of multistimulation with some shape were carried out. Based on the obtained experimental result, the appropriate tactile stimulus intensity and frequency for the recognition of multi-stimuli with some shape was discussed.

As a further study the additional experiment about investigating the appropriate and optimal number of tactels and spacing of the tactile stimulation array to enhance the tactile perception could be considered.

\section{ACKNOWLEDGMENTS}

This study was supported by a grant of the Korea Health 21 R\&D Project, Ministry of Health \& Welfare, Republic of Korea. (02-PJ3-PG6-EV100001)

\section{REFERENCES}

Bolanowski, S.J., G.A. Gescheider, R.T. Verrillo and C.M. Checkosky (1988). Four channels mediate the mechanical aspects of touch. Journal of acoustical society of America, Vol. 84, No. 5, pp. 1680-1694.

Caldwell, D.G., N. Tsagarakis and C. Giesler (2000). An integrated tactile/shear feedback array for stimulation of finger mechanoreceptor. Proc. of the 1999 IEEE international conf. on robotics \& automation, pp. 287-292.

Guyton, C. and J.E. Hall. (2002). Textbook of medical physiology, Elsevier science.

Johansson, R.S., U. Landström and R. Lundström (1982). Responses of mechanoreceptive afferent units in the glabrous skin of the human hand to sinusoidal skin displacements. Brain research. Vol. 244, pp. 17-25.

Konyo, M., S. Tadokoro and T. Takamor (2000). Artificial tactile feel display using soft gel actuators. Proc. of the 2000 IEEE international conf. on robotics \& automation, pp. 3416-3421.

Kontarinis, D., J. Son, W. Peine and R. Howe (1994). A tactile shape sensing and display system for teleoperated manipulation. IEEE International conf. on robotics \& automation, pp. 641-646.

Russell, R.A. (1990). Robot Tactile Sensing, Prentice hall.

Sato, K., E. Igarashi and M. Kimura (1991). Development of non-constrained arm with tactile feedback device. Proc. of the international conf. on advanced robotics, IEEE, pp. 334-338.

Shoval, S., I. Ulrich and J. Borenstein (2003). Robotics-based obstacle-avoidance systems for the blind and visually impaired. IEEE robotics \& automation magazine, pp. 9-20.

Watanabe, T. and S. Fukui (1995). A method for controlling tactile sensation of surface roughness using ultrasonic vibration. IEEE International conf. on robotics \& automation, pp. 1134-1139.

Yoon, M.J., T.K. Kwon, K.H. Yu and N.G. Kim (2004). Fabrication and evaluation of tactile stimulator array using stacked PZT. International conference on control, automation and systems, CD-ROM. 